\title{
Philosophiques
}

\section{Kant et la nature}

\section{Roberto Miguelez}

Volume 23, numéro 2, automne 1996

URI : https://id.erudit.org/iderudit/027395ar

DOI : https://doi.org/10.7202/027395ar

Aller au sommaire du numéro

Éditeur(s)

Société de philosophie du Québec

ISSN

0316-2923 (imprimé)

1492-1391 (numérique)

Découvrir la revue

\section{Citer cet article}

Miguelez, R. (1996). Kant et la nature. Philosophiques, 23(2), 253-264.

https://doi.org/10.7202/027395ar

\section{Résumé de l'article}

Le but de cet article est de repérer dans la Doctrine du droit, première partie de la Métaphysique des moeurs de Kant, ce qui peut bien être considéré comme une conceptualisation de la nature, et du rapport des humains à la nature, que la critique écologique contemporaine place au coeur même de la modalité pratique de la science et de la technologie modernes. Nous faisons en particulier référence au rapport d'appropriation de la nature dont la propriété n'est, chez Kant, dans ce contexte, qu'un corollaire et à la constitution du monde comme altérité absolue de la chose privée de tout droit, par rapport à laquelle il n'y a donc point d'obligation.
Ce document est protégé par la loi sur le droit d'auteur. L'utilisation des services d'Érudit (y compris la reproduction) est assujettie à sa politique d'utilisation que vous pouvez consulter en ligne.

https://apropos.erudit.org/fr/usagers/politique-dutilisation/ 


\title{
KANT Et LA NATURE
}

\author{
PAR \\ Roberto Miguel EZ
}

\begin{abstract}
RÉSUMÉ : Le but de cet article est de repérer dans la Doctrine du droit, première partie de la Métaphysique des mœurs de Kant, ce qui peut bien ètre considéré comme une conceptualisation de la nature, et du rapport des humains à la nature, que la critique écologique contemporaine place au cour mème de la modalité pratique de la science et de la technologie modernes. Nous faisons en particulier référence au rapport d'appropriation de la nature dont la propriété n'est, chez Kant, dans ce contexte, qu'un corollaire et à la constitution du monde comme altérité absolue de la chose privée de tout droit, par rapport à laquelle il n'y a donc point d'obligation.

ABSTRACT: The aim of this article is to identify in the Doctrine of Right of Kant's The Metaphysics of Morals the elements of what may be considered a conceptualisation of nature, and of the human relation to nature arguing that such a conceptualisation is precisely the object of contemporary ecological critique insofar as it animates modern scientific and technological practices. Particular reference is made to the relation of appropriation of nature of which private property is, in the context, but a corollary and to the constitution of the world as absolute otherness in relation to which there can be no question of obligation.
\end{abstract}

Nous pouvons situer chez Kant ce moment inaugural du transcendantalisme rationaliste, moment dans lequel la raison, au cours d'un processus d'auto-réflexion, se connait et reconnait comme pouvoir de construction de l'intelligibilité du monde. Pour la première fois, la raison ne s'épuise pas dans son exercice mais, dans et par un mouvement sur elle-même, le pense, et dans cette pensée découvre que les propriétés du monde ne peuvent être saisies si ce n'est que comme des propriétés pour une raison, et la raison elle-même, comme pouvoir configuratif des qualités du monde, de son intelligibilité.

Lorsque le transcendantalisme fait du sujet connaissant un sujet actif, producteur de déterminations cognitives, il prépare la voie à la conception du sujet comme sujet autrement actif, producteur d'objets et non seulement d'objectivités. Ce n'est, en effet, qu'à la suite de ce transcendantalisme purement gnoséologique que le pouvoir de donner un sens aux choses du monde peut se doubler du pouvoir de donner des choses au monde, de changer le monde des choses suivant les principes de la raison. L'instrumentalisation de la science 
de la nature réalise cette possibilité inhérente au transcendantalisme philosophique rationaliste, mais c'est encore chez Kant lui-mème que l'on peut trouver, bien que par une voie à première vue, et seulement à première vue, bien détournée, une de ces conditions de possibilité. Car c'est à propos de la propriété que cette condition se formule, donc en dehors aussi bien d'une thematisation de la raison pure que d'une thematisation de la raison pratique : dans la doctrine du droit.

Lorsque nous parlons d'instrumentalisation de la science de la nature nous faisons rëférence au fait que, bien qu'inspirée dans la tradition grecque de la théorie, la science moderne de la nature se donne comme visée la maitrise du monde des choses ${ }^{1}$. Cette visée suppose qu'un tel monde est effectivement constitué en tant que monde séparé du sujet - sans qu'il soit pour autant indépendant du sujet. Sociologiquement, elle suppose donc que la nature soit constituée dans et par la culture comme altérité absolue : objective et positive face à la subjectivité du sujet - ce que ni les sociétés "primitives " ni les sociêtés "traditionnelles" n'avaient fait ${ }^{2}$. Le présupposé juridique de cette constitution est l'idée moderne de la proprièté ou, pour parler plus exactement, l'idée que la propriété moderne permet de concevoir du rapport du sujet au monde des choses comme rapport d'altérité absolue. Or, c'est chez Kant que se trouve formulée d'une manière systématique, nous dirions même exemplaire, cette idée. Pour le montrer, nous nous tiendrons dans ce qui suit à un examen de certaines propositions contenues dans la Doctrine du droit, première partie de la Métaphysique des mœurs ${ }^{3}$.

\section{La propriété (juridique) comme possession intelligible}

Dans la première section de la première partie de la Doctrine universelle du droit, Kant envisage le problème fondamental de savoir

1. "The modern sciences of nature, though in part a legacy of Greek speculative curiosity, were tied inseparably to considerations of power and productivity. The clear primacy accorded by the ancients to theory over practice was reversed in modern intellectual culture. [...] The Greek identification of knowledge and virtue was transmuted by the moderns into a new identity of knowledge with power ". Michael McCarthy, The Crisis of Philosophy, State University of New York Press, New York, 1990, p. 3. C'est dans ce phénomène que McCarthy voit aussi un des éléments décisifs de la révolution scientifique moderne.

2. "[...] les sociétés primitives ne différencient pas catégoriquement, ontologiquement, les dimensions subjectives et objectives du rapport au * monde" (animisme). Une telle différenciation s'opère déjà dans les sociétès traditionnelles, sans y acquérir toutefois aucune valeur absolue [...]. Le monde empirique [...] intègre encore en lui ontologiquement une " aura " de subjectivité qui est essentiellement vécue d'un côté sur le mode esthétique, comme " harmonie", et de l'autre sur le mode normatif comme hiérarchie ontologique (la hiérarchie des " sphères " et des degrés de dignité des ètres, qui est aussi une hiérophanie ". Michel Freitag, "L'identité. l'altérité et le politique ", Sociétés, $n^{\circ} 9$, Hiver 1992, p. 18. J. Habermas avait déjà développé une idée semblable en utilisant la catégorie centrale de " décentration d'une compréhension egocentrée du monde " pour saisir le passage de la vision mythique à la vision moderne du monde. Voir Jürgen Habermas, Théorie de l'agir communicationnel, Paris, Fayard, 1987, vol. 1 . Intro., par. 2.

3. Tous les renvois se rapporteront à l'édition française préfacée par M. Villey. dont la traduction et l'introduction sont dues à A. Philonenko, publiée par la Librairie Philosophique J. Vrin à Paris, en 1971. (Citë dorénavant M.M.). 
comment on peut avoir quelque chose d'extérieur comme sien. Remarquons, d'emblée, que le problème concerne quelque chose d'extérieur (à moi) ${ }^{4}$ car, nous le verrons immédiatement, seule cette extériorité de la chose va permettre d'introduire la distinction philosophiquement cruciale entre possession sensible et possession intelligible.

Kant commence le traitement de ce problème avec une définition du mien selon le droit (meum iuris) :

Le mien selon le droit (meum uris) est ce à quoi je suis tellement lié, que l'usage qu'un autre en ferait sans mon agrément me léserait ${ }^{5}$.

Le mien selon le droit se laisse donc d'abord déterminer négativement, c'est-à-dire par rapport à ce qui n'est pas d'un autre, et subjectivement parce que, (a) la détermination de ce qui me léserait dans l'usage par l'autre me revient ; (b) c'est mon manque d'agrément qui constitue (au sens phénoménologique du terme) l'atteinte dont l'autre devient responsable ${ }^{6}$. Or, comme le signale Kant immédiatement, il serait contradictoire de dire que l'usage que quelqu'un fait d'une chose dont je suis pourtant en possession me lèse si le concept de possession était celui d'une possession physique ou sensible (detentio). Cependant, le mien selon le droit suppose que je puisse ètre lésé par l'usage qu'autrui fait d'une chose dont je ne suis pas en possession physique ou sensible. Ce qui veut dire qu'une autre forme de possession existe, qui plus est qu'elle correspond au concept du mien selon le droit, et qui s'oppose à la possession sensible. Kant l'appelle "possession intelligible ${ }^{7}$.

Un exemple est fourni par Kant lui-mème afin d'éclairer la distinction. Une pomme, dit-il, n'est pas mienne tout simplement parce que je la tiens dans ma main, c'est-à-dire parce que je la possède physiquement. Inversement, ce n'est pas parce que je laisse en tel ou tel endroit une pomme qui m'appartient qu'elle cesse, par là, de mappartenir. Le mien selon le droit, la possession juridique est donc une possession non sensible, "intelligible ". En fait, la distinction entre possession sensible et possession intelligible renvoie, ici, à la distinction entre usage effectif et pouvoir d'usage. En effet, une chose est mienne selon le droit non pas parce que j'en fais usage mais parce que j'ai le pouvoir d'en faire usage. Kant exprime cette idèe de la manière suivante :

[...] toutes les conditions de l'intuition, qui fondent la possession empirique, doivent ètre écartées (il faut les négliger) afin d'étendre le concept de la possession au-delà de celui de la possession empirique et de pouvoir

4. Ce qui ne veut pas dire que la question ne se pose pas de savoir si je me possède, et comment. Mais cette question n'est, chez Kant, pertinente que dans le contexte du problème du rapport de possession que d'autres peuvent entretenir avec moi et, par conséquent, pour lesquels je suis encore un objet extérieur, bien que nullement sous la modalité de la chose (purement corporelle). M.M., p. 121-122.

5. M.M., p. 119.

6. Est exclu donc d'emblée que le mien puisse coïncider avec le nôtre, que l'usage que l'autre fasse sans mon agrément puisse ne pas constituer une atteinte par rapport à moi, enfin que la détermination de l'atteinte puisse ètre objective et non pas subjective - par exemple, le produit d'une délibération. Nous reviendrons sur les considérations de Kant sur le nôtre.

7. Le terme est introduit dans le mème par. 1 (p. 119). 
dire : tout objet extérieur de l'arbitre que j'ai en ma puissance (Gewalt) (et dans la mesure seulement où je l'ai en ma puissance), sans en ètre en possession peut être compté comme juridiquement mien ${ }^{8}$.

D'ailleurs, si la possession physique (sensible ou empirique), c'est-à-dire l'usage effectif que je fais de la chose était un critère nécessaire, voire suffisant, il ne serait pas possible, par exemple, de posséder (en droit) deux choses qui se trouvent éloignées dans l'espace et/ou dans le temps. C'est pourquoi la notion de "détention" (detentio) doit laisser sa place, dans le droit, à la notion d' avoir" qui " fait abstraction de toutes les conditions spatio-temporelles, et qui ne suppose rien, si ce n'est que l'objet soit en ma puissance (in potestate mea positum esse $)^{9}$ "- la puissance étant conçue par Kant comme la possibilité (juridique) de ma volonté de faire de la chose l'usage qui lui convient ${ }^{10}$.

La distinction entre possession sensible et possession intelligible permet à Kant d'associer la première à la possession dans le phénomène (possessio phænomenon), et de considérer la deuxième comme "possessio noumenon". Certes, du cōté de la possessio phænomenon la symétrie n'est pas exacte avec l'Analytique transcendantale car, comme Kant lui-mème le signale, l'objet possédé sensiblement n'est pas considéré tout simplement comme phénomène mais bel et bien comme chose en soi ${ }^{11}$. Mais du côté de la possessio noumenon nous sommes censés nous trouver, par contre, dans une situation symétrique : "Le droit, dira Kant, est un pur concept pratique rationnel de l'arbitre sous des lois de la libertét ${ }^{12}$ ". Or, dans la mesure où la possession intelligible ("l'avoir") relève du juridique, le juridique apparait relié au noumenon. Davantage, puisque la possession intelligible est une création du juridique, c'est le juridique qui constitue, dans cette dimension pratique, le noumeron lui-même. C'est pourquoi aussi le droit peut être caractérisé comme "pur concept pratique rationnel ", bien que le statut de la rationalité soit ici, à la différence des concepts purs de la raison pure, double, si l'on peut parler ainsi.

En effet, d'une part, ce n'est que gràce au concept de la possession intelligible que la propriété (juridique) peut être saisie dans ses déterminations nécessaires, c'est-à-dire selon sa définition réelle $^{13}$ mais, d'autre part, la propriété juridique elle-méme n'est qu'une création du juridique de sorte que l'assimilation par Kant de la propriété à la possessio noumenon a comme effet - et comme présupposé - d'assimiler rationalité et juridicité. Kant le dit luimème, d'ailleurs: "Toutes les propositions juridiques sont des propositions a priori, car elles sont des lois rationnelles (dictamina rationis) ${ }^{14}$ ".

8. M.M., p. 126.

9. M.M., p. 127.

10. M.M., p. 128.

11. M.M., p. 123.

12. lbid.

13. Voir Ibid.

14. M.M., p. 124. Cette conception se trouve déjà chez Leibniz qui écrit, par exemple : "La doctrine du droit est du nombre de celles quil ne dépendent pas des expériences mais des définitions, ni des démonstrations à partir des sens mais à partir de la raison et qui pour ainsi dire sont de droit et 
Nous proposons de retenir de ce premier examen les trois points suivants : 1) la priorité logique de la possession individuelle (du " mien $"$; 2) la substitution de la puissance d'usage à l'usage effectif comme critère de la possession; et 3 ) l'assimilation du juridique et du rationnel. Nous reviendrons en particulier sur le premier de ces trois points.

\section{La séparation ontologique du mien}

La question du statut de la nature dans la Doctrine du droit présuppose une véritable séparation ontologique du mien (extérieur) que Kant s'empresse d'effectuer dès les premiers paragraphes de la première partie de la Doctrine:

Les objets extérieurs de mon arbitre ne peuvent être que de trois sortes: 1. une chose (corporelle) en dehors de moi ; 2 . l'arbitre d'un autre relativement à une action (tat) déterminée (præstatio) ; 3. l'état d'un autre par rapport à moi $[\ldots]^{15}$.

Cette classification suppose, en effet, la division de la réalité telle que constituée dans et par le droit, en une réalité de choses et une réalité de personnes. Si nous pouvons posséder et des choses et des personnes, ce ne le sera pas pourtant de la mème manière en raison de cette différence ontologique. À son tour, cette réalité de personnes peut appartenir au mien de deux manières: sous la forme du service ou de la prestation, et sous la forme de l'" état". C'est sous cette dernière forme que Kant envisage le mien par rapport à la femme, aux enfants et aux serviteurs ${ }^{16}$. Aussi, et en correspondance avec cette classification et la séparation qu'elle suppose, le champ du droit (privé) s'organisera autour de modalités diverses : le droit réel, le droit personnel, le droit domestique.

Mais ce n'est pas arbitraire ou fortuit que la classification de ces objets extérieurs commence par les choses. En effet, même s'il existe une séparation ontologique entre les choses et les personnes, les " catégories " de la possession et, tout particulièrement, celle de la puissance d'usage, sont également applicables - quoique non pas de la même manière ou avec les mêmes conséquences ${ }^{17}$. Il n'en demeure pas moins qu'il est possible de conclure à un rôle en quelque sorte

non de fait". Leibniz, Elementa juris naturalis, dans Leibniz. Le droit de la raison, Textes réunis et présentés par René Sève, Librairie philosophique Vrin, Paris, 1994 , p. 90.

15. M.M., p. 121 .

16. La notion d' "état " (Zustand) renvoie à l'idée d'un rapport hiérarchisé - et non pas, comme dans le cas de Stand, à l'idée de statut (social).

17. Par exemple, l'application de cette "catégorie " au troisième " objet extérieur de mon arbitre", à savoir cet "état "d'un autre créé par le mariage conduit, tout au début de l'exposition du droit conjugal, à une définition de la "communauté sexuelle" comme "usage réciproque qu'un homme peut faire des organes et des facultés sexuels d'une autre personne" (M.M., p. 156). La réciprocité dans l'usage fait du rapport des époux * un rapport d'égalité de possession " (M.M., p. 157) qui, bien entendu, n'est pas concevable chez Kant entre l'homme et la nature. La séparation ontologique entre choses et personnes rend asymétrique le rapport institué par la catégorie de la puissance d'usage - tandis que l'appartenance ontologique au monde des personnes le rend symétrique, au moins en principe. 
paradigmatique du rapport (juridique) aux choses ${ }^{18}$. C'est dans l'élucidation de ce rapport qu'apparait dans la Doctrine du droit le thème de la nature - certes, sous la forme de monde extérieur de choses et, en particulier, sous le thème du sol ou de la terre.

\section{Le rapport à la nature}

Dans la Doctrine du droit, la question du rapport à la nature est traitée, pour l'essentiel, sous deux aspects: l'un que l'on pourrait appeler "transcendantal " parce qu'il réfère aux présuppositions de la raison, l'autre qui déplace la référence au juridique. Ces deux aspects se trouvent cependant articulés, le juridique étant subordonné à l'aspect "transcendantal " de la question. Nous commencerons par celui-ci.

Le deuxième paragraphe de la Doctrine $d u$ droit porte sur le "Postulat juridique de la raison pratique". Deux propositions de ce paragraphe méritent d'ètre examinées. La première porte sur le rapport aux choses, la deuxième sur le rapport entre les personnes tel qu'institué, par le droit, à partir du rapport aux choses. Voici la première proposition :

[...] un objet de mon arbitre est ce dont j'ai la faculté physique de faire tel ou tel usage, ce dont l'usage est en mon pouvoir (potentia) (Macht) ; [...] pour ne faire que penser quelque chose en tant qu'objet de mon arbitre, il suffit que j'ai conscience qu'il est en mon pouvoir (potentia-Macht). C'est donc une présupposition a priori de la raison pratique que de considérer et de traiter tout objet de mon arbitre comme étant objectivement un mien et un tien possibles ${ }^{19}$.

Nous retrouvons ici la catégorie fondamentale de la puissance d'usage dans la définition mème de l'objet de mon arbitre, cependant la définition ne sert pas seulement à concevoir la possession d'un objet (l'objet de mon arbitre est un mien possible), elle sert aussi à concevoir la "possessibilité "de tout objet pour autant que puisse s'exercer sur lui ma puissance d'usage (tout objet de mon arbitre est un mien possible). Qui plus est, le postulat juridique de la raison pratique fonde sur la liberté de l'être humain ce rapport d'appropriation de tout objet extérieur :

[...] si juridiquement quelque chose ne pouvait absolument pas ètre en mon pouvoir, c'est-à-dire que si en faire usage ne pouvait ètre compatible avec la liberté de chacun suivant une loi universelle (si cet usage était injuste), alors la liberté se priverait elle-mème de l'usage de son arbitre par rapport à un objet de celui-ci, par le fait mème qu'elle mettrait des objets inutilisables

18. Ce rôle paradigmatique du rapport aux choses, surtout en ce qui concerne l'application aux personnes de la catégorie de la puissance d'usage peut comporter, mème en tenant compte de l'asymétrie à laquelle nous venons de nous référer, des conséquences certainement non négligeables. Nous pensons à la réification des rapports humains que la définition kantienne de la communauté sexuelle suppose, et que Kant lui-même reconnait, sans lui donner d'autre solution que celle, bien discutable, de dissoudre la réification dans la réciprocité du devenir chose - l'un pour l'autre. (Voir, à cet égard, le par. 25 du Titre premier du Droit domestique). Mais c'est une question que nous n'examinerons pas ici.

19. M.M., p. 120-121. 
hors de la possibilité de tout usage, c'est-à-dire qu'elle les anéantirait à um point de vue pratique et les définirait comme res nullius ${ }^{20}$.

$\mathrm{Si}$ nous nous sommes permis de transcrire in extenso cet argument de Kant c'est parce qu'il montre d'une manière on ne peut plus claire comment le postulat juridique de la raison pratique fonde sur la liberté de l'être humain conçue comme liberté d'usage, le rapport décisif d'appropriation des choses, c'est-à-dire de la nature. Dès lors, l'arbitre humain ne consiste qu'à rendre tout objet susceptible d'appropriation ou, en d'autres termes, à soumettre le monde des objets, la nature, à la puissance de détermination pratique des sujets.

La deuxième proposition qui mérite d'être examinée est la suivante :

On pourrait appeler ce postulat une loi permissive (lex permissival de la raison pratique, qui nous donne une faculté (Befugnis) [...] je veux dire la faculté d'imposer à tous les autres une obligation, qu'ils n'auraient pas autrement : celle de s'abstenir d'user de certains objets de notre arbitre, parce que nous les avons d'abord pris en notre possession ${ }^{21}$.

Si, donc, en vertu de la proposition antérieure tout objet peut, en principe, etre objet d'appropriation, en vertu de cette deuxième proposition la seule restriction à l'appropriation découle du rapport avec les autres sujets. En effet, c'est parce que je me définis comme (premier) possesseur face aux autres que découle l'obligation pour ceux-ci de s'abstenir d'employer leur puissance d'usage sur l'objet de mon arbitre ${ }^{22}$. Il en découle aussi, et c'est une conséquence fondamentale pour la question que nous examinons, que, dès lors, les choses n'ont pas de droits en elles-mèmes - plus exactement, elles n'ont pas de droits tout court - le droit n'étant valable que pour des rapports entre personnes - dans ce cas, vis-à-vis des choses.

Que le droit ne vaut qu'entre personnes, c'est-à-dire qu'il appartient à la région ontologique des ètres humains, cette proposition peut constituer une évidence ${ }^{23}$. Mais au-delà ou en deçà de la catégorie du droit comme institution (juridique) présupposant une

20. M.M., p. 120.

21. M.M., p. 121 .

22. Ces deux propositions reviennent plus loin dans le texte que nous commentons. La première est formulée ainsi : *[...] il revient à chacun selon le postulat de la raison pratique la faculté d'avoir un objet extérieur de son arbitre comme sien - que, par conséquent, toute détention est un état dont la légitimité se fonde sur ce postulat par un acte antérieur de la volonté ". La deuxième est formulée ainsi : "Cet acte, si une possession plus ancienne du mẻme objet par un autre ne s'y oppose, m'autorise, provisoirement, selon la loi de la liberté extérieure, à interdire à quiconque [...] toute prétention à user de cet objet [...] \%. M.M., p. 132 .

23. Peut mais ne l'est pas toujours, la preuve en est que, chez Marcuse par exemple, la nature semble sinon avoir des "droits "au moins pouvoir être, davantage devoir être l'autre d'un rapport autre que celui d'une attitude purement "technique", à savoir le partenaire (Gegen-spieler) dans une interaction possible. (Voir, en particulier, Herbert Marcuse, One Dimensional Man. Boston. Beacon Press, 1966). Rappelons que J. Habermas, déjà dans La techrique et la science comme *idéologie", critique cette conception de Marcuse qui aboutirait à effacer la distinction, fondamentale pour Habermas, entre travail et interaction, entre nature et société. Voir J. Habermas, La technique et la science comme " idéologie ", Paris, Gallimard, 1973, p. 11 ss. 
inter-subjectivité on pourrait dire que, chez Kant, les choses n'ont pas de "droits" non pas seulement parce qu'elles ne font pas partie du mème univers ontologique que les ètres humains - elles ne sont pas des sujets - mais encore parce qu'elles sont entièrement et absolument soumises à la puissance d'usage des êtres humains. Kant laffirme dans les termes suivants :

L'objet extérieur qui selon la substance est le mien de quelqu'un, est la propriété (dominium) de celui auquel tous les droits sur cette chose sont inhérents [...]. Mais en conséquence il va de soi qu'un tel objet ne saurait ètre qu'une chose corporelle envers laquelle on n'a point obligation $[\ldots]^{24}$.

Si la première section de la première partie de la Doctrine porte sur la manière d'avoir quelque chose d'extérieur comme mien, la deuxième section de cette partie porte, elle, sur la manière d'acquérir quelque chose d'extérieur. C'est dans cette partie que se précise le rapport à la nature comme un type particulier de rapport de propriété qui découle de, ou implique une relation complexe entre avoir et acquérir. Dans le traitement de cette relation, la distinction entre communauté " originaire " et communauté "primitive "du sol joue un rôle décisif.

Comment saisir la "dialectique "entre ces deux formes de communauté ? Pour Kant, la propriété primitive du sol, si elle était considérée comme empiriquement première, ne pourrait être qu'une fiction, voire une contradiction dans ses termes. En effet, pour ètre première empiriquement, elle aurait dû être instituée, et cette institution ne pourrait ètre que celle découlant d'un contrat en vertu duquel chacun aurait renoncé à sa propriété au bénéfice de la possession commune. Ainsi donc il y aurait contradiction dans la notion même de communauté primitive ainsi comprise. Pourtant, pour que chacun ait pu s'approprier des choses du monde, il a fallu que tous possèdent le monde, cette possession devant alors être considérée comme une condition non pas empirique, mais trancendantale. Écoutons Kant :

[...] la possession de tous les hommes sur la terre, qui précède tout acte juridique de leur part [...] est une possession commune originaire [...] dont le concept [...] est un concept pratique rationnel, qui contient a priori le principe seul d'après lequel les hommes peuvent faire usage [...] de leur lieu (Platz) sur la terre ${ }^{25}$.

Bien entendu, pour que la simple possession physique des choses, c'est-à-dire de la nature puisse constituer déja un droit il faut soit que tous puissent, en principe, c'est-à-dire transcendantalement, disposer de la nature et, donc, en avoir la propriété en commun en tant que propriété originaire (communio mei et tui originaria), ou soit que la nature n'appartienne à personne - tout en pouvant, en principe, appartenir à chacun. Kant élimine cette possibilité puisque, "originairement (c'est-à-dire avant tout acte juridique) tous les hommes sont "déjà " en une possession du sol"

24. M.M., p. 146. C'est moi qui souligne. R.M.

25. M.M., p. 138-139.

26. M.M., p. 138. Si nous utilisons les paragraphes $6,250,18$ à 251,36 qui se trouvent, autant dans la traduction d'A. Philonenko que dans celle d'Alain Renaut dans la première section de la Métaphysique des moeurs, nous 
remarquons qu'il manque aussi une troisième possibilité à laquelle Kant ne réfléchit pas, à savoir que la nature s'appartienne à ellemème. Et pour cause, pourrait-on dire, car de ce silence pointe l'idée fondamentale suivant laquelle une nature qui serait libre, c'est-à-dire sans possesseur, interdirait transcendantalement à chacun de s'en approprier. Autrement dit, une nature qui serait libre annulerait la liberté des ètres humains.

D'une certaine manière, et plutôt implicitement, la distinction entre possession sensible et possession intelligible trouve ici, c'està-dire à propos de la manière d'acquérir quelque chose d'extérieur, son équivalent dans la distinction entre communauté primitive et communauté originaire de la nature. Toutefois, tandis que la possession intelligible renvoie au juridique, la communauté originaire renvoie au transcendantal. La conséquence est non seulement que la première possession sensible est déjà un "droit "- non pas, certes, au sens juridique puisqu'il n'y a pas encore de "juridique "mais en vertu des postulats mêmes de la raison pratique - mais encore, et dans les termes mêmes de Kant, que "la première acquisition d'une chose ne peut ètre que celle du sol ", c'est-à-dire de la nature ${ }^{27}$.

Il est important de signaler que lorsque Kant se réfère à la première possession sensible et au droit qu'elle crée déjà en tant que telle, il exclut explicitement le travail - le premier travail ou, en général, "la première transformation"- comme titre de l'acquisition. D'une part, pour Kant le travail porte non pas sur la "substance " mais sur les " adhérences" ou "accidents", tandis que la possession porte sur la "substance". Mais, d'autre part, ce n'est pas le travail qui définit essentiellement notre rapport aux choses mais la puissance d'usage ${ }^{28}$. Le thème d'une "construction " de la nature dans et par le travail humain qui, on le sait, ouvre à la réflexion un vaste champ de possibilités philosophiques se trouve déclassé au profit d'une thématique qui pointe plutôt vers la "construction " de soi-mème ${ }^{29}$.

Enfin, il est encore important de remarquer que, pour Kant, la finitude de la terre joue un rôle déterminant dans la formation de la

retrouvons cette impossibilitê exprimée de la manière suivante: "Le possesseur se fonde sur la propriété innée commune du sol, et sur la volonté universelle a priori accordée avec celle-là qui permet une possession particulière sur elle lcar autrement les choses vacantes seraient érigées en soi et d'après une loi en choses sans possesseur (herrenlos) [...] ". M.M., p. 124-125. Aussi Kant, Métaphysique des moeurs. II, traduction, présentation, bibliographie et chronologie par Alain Renaut, Paris Flammarion, 1994.

27. M.M., p. 137. En fait Kant restreint la notion du sol à "toute partie de terre qui est habitable " et fait une distinction entre le sol et ce qui se meut sur lui mais ce n'est que pour régler la question de l'appropriation des choses "mobiles "qui se trouvent sur la terre.

28. M.M., p. 145. Dans cette "Remarque " au par. 17 consacré à la question du travail Kant revient sur la proposition fondamentale suivant laquelle " le droit par rapport à tout possesseur d'une chose ne signifie que la facultè de l'arbitre particulier de faire usage d'un objet ".

29. D'un autre côté, comme le remarque A. Renaut, Kant prend ici "le contrepied parfait de la thèse défendue par Fichte en 1793 dans les Contributions destinées à rectifier l'opinion du public sur la Révolution française et réaffirmée par lui en 1796 dans le Fondement du droit naturel [...] - savoir la légitimation de la propriété d'un objet par référence au travail incorporé en lui ". Alain Renaut, ibid, p. 382. 
communauté humaine $^{30}$ et donc, par là-même, dans l'émergence du juridique. Le raisonnement qui sous-tend cette proposition fait de la finitude un antécédent du " droit "qu'est la première possession sensible, et de ce "droit "l'antécédent du juridique en tant que droit au sens plein du terme, c'est-à-dire au sens d'acte (juridique) de l'arbitre $^{31}$. En d'autres termes, si c'est la finitude qui crée la propriété, $c^{\prime}$ est la propriété qui engendre le juridique ${ }^{32}$.

Si l'idée suivant laquelle la finitude du monde engendre la socialite $\dot{e}^{33}$ est, à proprement parler, une idée étonnante - surtout à la lumière des conceptions classiques de la philosophie politique comme celles d'un Aristote ou d'un Hobbes -, l'idée suivant laquelle la propriété engendre le juridique l'est bien moins. Elle se suit en toute logique de l'idée de possession ou de propriété comme interdiction, exclusion - idée qui, on se rappelle, se trouve au début mème du traitement de la question du mien. En effet, si la terre n'était pas finie, c'est-à-dire si les ressources étaient illimitées, il n'y aurait pas possibilité de compétition et, donc, nécessité d'exclusion. L'idée de propriété renvoie ainsi à l'idée fondamentale de l'économie politique classique suivant laquelle les ressources sont "rares", c'est-à-dire limitēes.

Nous dégageons de la Doctrine du droit une dernière proposition cruciale pour notre propos. Nous nous permettrons encore de nous référer directement à Kant :

$J$ 'acquiers quelque chose quand je fais (efficio) que quelque chose devienne mien. [...]. Le principe de lacquisition est le suivant : ce que je soumets à ma puissance (suivant la loi de la liberté externe) et dont j'ai la facultê d'user comme d'un objet de mon arbitre (d'après le postulat de la raison pratique), enfin, ce dont je veux (conformément à l'idée d'une volonté unifiée possible) qu'il soit mien : cela est mien ${ }^{34}$.

Le " je " qui revient sans cesse dans ce texte choisi du début de la deuxième section de la Doctrine marque clairement le rapport originaire au monde et à la nature comme étant un rapport individuel : seuls des individus font face au monde. Pour qu'il ne demeure aucun doute quant à ce rapport, Kant réaffirmera immédiatement que "l'état de communauté du mien et du tien

30. * [...] si la terre était une plaine indëfinie les hommes pourraient tellement s'y disperser, qu'ils n'en viendraient jamais à former entre eux une communauté, et ainsi la communauté ne serait pas une nécessaire conséquence de leur existence sur la terre ". M.M., p. 138.

31. En toute rigueur, ce n'est pas seulement la finitude de la terre qui constitue un antécédent du " droit " de la première possession sensible mais aussi la multitude humaine : un homme qui serait seul sur la terre n'aurait pas à déclarer quelque chose comme sien (voir M.M., p. 137) - bien que, on devrait ajouter, il pourrait se déclarer possesseur de toutes les choses.

32. Il est aussi intëressant de signaler que dans Le Capital Marx s'attache à montrer que c'est plutōt l'inverse qui se produit en fait. On le voit en toute clarté là où, comme dans les Amériques, la terre est pratiquement - infinie "et la population très peu nombreuse. Le juridique intervient alors pour créer la propriété. C'est, pour Marx, le grand mérite de E.G. Wakefield de l'avoir découvert - et au Canada. Karl Marx, Le Capital, Livre premier, chap. XXV : La théorie moderne de la colonisation.

33. Certes, non pas la socialité "naturelle " des couples ou de familles.

34. M.M., p. 133 . 
(communio) ne peut jamais ètre pensé comme originaire ${ }^{35}$. Or, ce principe individualiste renvoỉe aussi immédiatement à la volonté de l'individu conçue comme volonté de puissance. Du même coup, l'acquisition - ou l'appropriation - se définit comme soumission - à ma volonté de puissance. L'usage que je fais alors de la chose ne suppose aucun respect de la chose - aucune obligation par rapport à la chose, comme nous l'avons déjà vu - car elle est tout entière soumise à ma volonté.

\section{Conclusion}

Si dans la perspective cognitive du transcendantalisme rationaliste, l'intelligibilité du monde trouve ses conditions de possibilité chez le sujet, il en va de même dans la perspective pratique de ce transcendantalisme. Mais à la différence de l'appropriation cognitive du monde, lappropriation pratique saisit le monde non seulement comme phénomène mais, on l'a vu, bel et bien comme chose en soi. Seulement, cette chose en soi, loin d'être pour soi, l'est pour le sujet. En fait, c'est dans lappropriation pratique du monde que le monde s'avère un monde pour le sujet, c'est-à-dire entièrement soumis aux déterminations de celui-ci.

C'est dans la liberté du sujet, qui s'exprime dans sa volonté de soumettre le monde, et qui fait de cette soumission du monde une condition pratique de sa liberté, que le monde apparait dans l'altérité absolue de la chose privée de tout droit, par rapport à laquelle il n'y a donc point d'obligation. Face à la puissance d'usage du sujet, la nature ne se définit que comme nature utilisable. La propriété n'est que le corollaire d'un rapport à la nature qui est, par principe, un rapport d'appropriation. Il est ainsi possible de voir à l'œuvre dans la Doctrine du droit de Kant une conceptualisation de la nature et du rapport des humains à la nature que les débats écologiques contemporains placent au cœur de la modalité pratique de la science et de la technologie modernes.

Lorsque nous parlons d'écologie nous ne nous référons pas à cette branche de la biologie qui s'occupe du rapport des organismes vivants à leur environnement - sens qui se trouve enregistré, jusqu'à présent, dans les dictionnaires - mais, plus précisément, à cette perspective qui examine les conséquences sur la nature de certaines pratiques modernes, spécialement des pratiques économiques de production et de consommation dont la modalité et l'universalité ont été rendues possibles par la science et la technologie. Cette perspective écologique bien récente - car elle s'est développée dans les trente dernières années - présente des différences de taille avec celle, traditionnelle, associée à la biologie. En effet, d'une part, la perspective écologique contemporaine se veut critique et, par conséquent, se dissocie d'une neutralité axiologique attribuée traditionnellement aux sciences. D'autre part, elle ne manque pas de

35. Ibid. C'est pourquoi, comme le signale $\mathrm{A}$. Renaut - bien que dans une note portant sur un autre paragraphe - * la supposition. purement transcendantale, de la communauté originaire du sol n'a donc rien à voir avec l'utopie d'un communisme primitif qui eút pu exister : Idée de la raison, la communauté onginaire est la condition de pensabilité du droit privè ». A. Renaut, ibid, p. 381. 
réfléchir aux présupposés anthropologiques et ontologiques des pratiques économiques modernes et du soubassement scientifique et technologique de celles-ci. Parmi ces présupposés, l'écologie contemporaine trouve une relation à la nature essentiellement utilitaire liée mème, dans certaines variantes de la critique écologique, à une relation sociale de domination ${ }^{36}$. C'est en ce sens précis que la conception de Kant de la nature comme domaine des moyens au service des finalités du seul sujet concevable, le sujet humain, exprime ce présupposé que la critique écologique trouve au cœur de la science et de la technologie modernes ${ }^{37}$.

Département de philosophie et Faculté des sciences sociales Université d'Ottawa

36. Pour une analyse des paradigmes écologiques contemporains et de leurs présupposés anthropologiques voir Koula Mellos, Perspectives on Ecology, McMillan, London, 1988.

37. Nous n'examinons pas ici car elle mériterait une analyse particulière la conception de Kant de ces " objets * naturels que seraient les animaux. (Voir, à cet égard, Alexander Broadie et Elizabeth Pybus, "Kant's Treatment of Animals *. Philosophy. The Joumal of the Royal Institute of Philosophy. October 1974, vol. 49, n 190. 